\title{
Polyunsaturated fatty acids and gene expression in mammalian systems
}

\author{
Klaus W. J. Wahle ${ }^{1 *}$, Dino Rotondo ${ }^{2}$ and Steven D. Heys ${ }^{3}$ \\ ${ }^{1}$ Rowett Research Institute, Bucksburn, Aberdeen AB21 9SB, UK \\ ${ }^{2}$ Department of Immunology, Strathclyde University, Glasgow G4 ONR, UK \\ ${ }^{3}$ Department of Surgical Oncology and Surgical Nutrition, Aberdeen University Medical School, \\ Aberdeen AB25 9ZD, UK
}

\begin{abstract}
Over the last 30 years it has become apparent that specific dietary fatty acids are capable of regulating, either directly or indirectly through various signal pathways, the expression of numerous genes, either positively or negatively. Such nutrient-gene interactions have important effects on cell metabolism, differentiation and growth, and ultimately on disease processes. The present review describes some of the more important fatty acid-gene interactions in relation to health and disease in mammalian species, and focuses on the underlying cell signal mechanisms, including various transcription factors, affected by fatty acids and some of their oxygenated derivatives, e.g. the eicosanoids. The review also attempts to clarify some of the complexities of the effects of fatty acids by suggesting a possible overriding regulation by the redox status of the cell. The latter will at least stimulate controversy in this exciting area of lipid research.
\end{abstract}

Nutrient-gene interactions: Dietary fatty acids: Eicosanoids: Cell signal mechanisms: Cell redox status

Fat is a vital component of the diet of all mammals, including man, despite the current fashion to portray it as the 'bête noir' of human nutrition that is responsible for all common human diseases. Its energy density makes it the most efficient form of energy storage known to biology that, in many feral animals, is an important survival mechanism in times of food deprivation. In industrialised societies, where food excess rather than deficiency is prevalent, this primordial survival mechanism is abused, resulting in obesity and its attendant health consequences.

Our understanding of the complex role of dietary fat, particularly the role of the specific components of the fatty acids in mammalian growth, development and health, has evolved rapidly in recent years. Far from being merely an energy store, important as that is, fatty acids derived from the diet are vital structural components of all cell membranes in the form of the various amphiphilic phospholipids that constitute the lipid bilayers. They are the basal lipid matrix without which cells could not exist. Cellular compartmentalisation and structural complexity, which has been instrumental in the evolution of life, is dependent on the semipermeable membranes represented by the lipid bilayer. Many types of fatty acid, including saturated, monounsaturated and polyunsaturated (PUFA) forms can play important structural and physico-chemical roles in cell membranes. The presence in membranes of different proportions of fatty acid types alters membrane fluidity, a physical characteristic that can modulate the activity of a myriad of proteins (enzymes, receptors, ion channels) that are incorporated into the lipid matrix of the membrane bilayers of all cells and are the biologicallyactive elements involved in cell functions (Gurr \& Harwood, 1991).

Two specific types of PUFA, i.e. LA (LA; $18: 2 n-6)$ and $\alpha$-linolenic acid (18:3n-3), are termed essential fatty acids because they cannot be produced in the mammalian body and need to be ingested from the diet. Their essentiality appears to reside predominantly in the capacity of their longer-chain derivatives dihomo- $\gamma$-linolenic acid (20 : $3 n-6)$; arachidonic acid (ARA, $20: 4 n-6$ ) and eicosapentaenoic acid (EPA, $20: 5 n-3)$ to act as precursors of hormone-like molecules termed eicosanoids. These eicosanoids (prostaglandins (PG), leukotrienes, thromboxanes, hydroxyeicosatetraenoates, hydroperoxyeicosatetraenoates) are oxidation derivatives of the fatty acids, and they represent yet another level of complexity and functionality of fats in mammalian biology in that they are potent signalling molecules that are involved in the regulation of

\footnotetext{
Abbreviations: ARA, arachidonic acid; CLA, conjugated linoleic acids; DHA, docosahexaenoic acid; EPA, eicosapentaenoic acid; FAS, fatty acid synthetase;

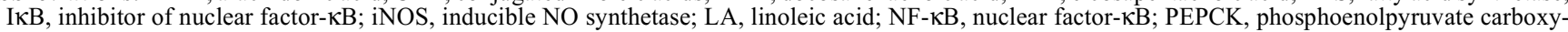
kinase; PG, prostaglandins; PPAR, peroxisome proliferator-activated receptors; PUFA, polyunsaturated fatty acids; SCD, stearoyl-CoA desaturase; SREBP, sterol regulatory element-binding protein.

*Corresponding author: Professor K. W. J. Wahle, fax +44 1224 715349, email k.wahle@rowett.ac.uk
} 
numerous cellular processes (Gurr \& Harwood, 1991; Funk, 2001).

Over the past 30-40 years another very important role of fat has gradually been elucidated. It has become increasingly apparent that dietary fats are capable of regulating, either directly or indirectly, the expression of numerous genes, both positively and negatively. Dietary fatty acids and their metabolic derivatives can modulate the responses of the cellular 'control centre', the genome, to physiological stimuli. Clearly, such nutrient-gene interactions will have important effects on metabolism, cellular differentiation and functions, growth, development and the health and well-being of mammalian species, including man (Jump \& Clarke, 1999; Duplus et al. 2000; Clarke, 2001). The present review is not comprehensive, but will describe what are considered as some of the more important aspects of fatty acid-gene interactions and will focus on the underlying mechanisms and the possible health implications of such interactions. Readers are directed to the excellent reviews cited for more comprehensive and detailed information on this fascinating topic.

\section{Early observations}

Approximately 40 years ago it was observed that the capacity of mouse liver to synthesise long-chain fatty acids de novo was greatly enhanced and the activities of some of the enzyme responsible (fatty acid synthetase (FAS), malic enzyme, glucose-6-phosphate dehydrogenase) were increased when fats containing LA were deficient in their diets. This observation indicated a negative regulatory influence of LA (Allman \& Gibson, 1969). Feeding fats containing LA, but not saturated or monounsaturated fat and cholesterol, reduced the increased fatty acid synthetic capacity induced by high-carbohydrate diets. Similar, but less clear changes in fatty acid synthesis were also observed in epididymal fat pads from these animals, suggesting that the LA effect was not limited to the liver. The authors suggested two mechanisms to explain the increased activity of hepatic and epididymal fatty acid-synthesising enzymes in high-carbohydrate-fed animals: a decrease in cellular free fatty acids or fatty acyl-CoA that would nonspecifically inhibit enzyme activity; an increase in the amount of enzyme protein produced as an adaptive response to the diet. The latter suggestion implied that the presence of LA would inhibit this adaptive response in enzyme synthesis. This hypothesis was not verified by the authors and no mechanistic explanation was given for their assumptions.

Subsequent studies verified the reduction in liver lipogenic enzyme activity when rats were fed diets containing $60 \mathrm{~g} \mathrm{LA} / 100 \mathrm{~g}$ total fat (Flick et al. 1977; Jeffcoat $\&$ James, 1978). Similarly, when rat pups are weaned from a high-fat diet (mothers milk) to a low-fat laboratory diet the protein expression of liver lipogenic enzymes is increased (Clarke et al. 1990). Wahle \& Radcliffe (1977) observed that when genetically-obese $(f a / f a)$ rats, which have an excessive genetically-determined lipogenesis, were fed a high-sunflower-seed oil diet high in LA content the characteristic hepatic lipid accretion, high fatty acid synthesis de novo and stearoyl-CoA desaturase (SCD) 1 activity were decreased by about $40-50 \%$ in comparison with standard diets and low-sunflower oil diets. The authors suggested that the sunflower oil diet was able to suppress the genetically-determined increase in lipogenesis in the falfa rats. The high-sunflower oil diets also reduced the activity of these lipogenic enzymes in the lean phenotypes $(F a / F a)$. It is thought that the effects were exerted at the level of the enzyme protein (catalytic efficiency) or its expression.

Schwartz \& Abraham (1982) investigated the regulation of FAS by LA in mouse liver in more detail and observed that the increase in FAS associated with high-carbohydrate diets was due to an increased hepatic content of the enzyme (specific antibody assay). This effect was due to an increase in the rate of de novo synthesis of the enzyme protein and a decrease in the rate of its degradation when compared with maize-oil-fed animals and not to a change in catalytic efficiency. Clearly, feeding maize oil reduced the synthesis and increased the degradation of FAS protein in mouse liver. These authors further demonstrated that the LA effect in inhibiting FAS synthesis was not dependent on its conversion to prostaglandins (PG). They observed that columbinic acid (trans-5, cis-9, cis-12-18:3), a PUFA that cannot act as a PG precursor, was as effective as the PG-precursor fatty acids (linolenic acid, LA and ARA) in reducing the hepatic content of FAS. This finding further supported the lack of PG involvement in decreased FAS protein synthesis. It is apparent from these findings that both $n-6$ and $n-3$ PUFA are equally capable of reducing FAS in rodent liver. The authors suggested that the ability of fatty acids to inhibit increased hepatic FAS protein depended on the extent of unsaturation and the position of individual double bonds in the acyl chain. Subsequent studies have shown that this suggestion is an oversimplification and that fatty acids, their CoA derivatives and various oxidation products, including eicosanoids, can regulate the protein content of numerous enzymes involved in the homeostatic control of carbohydrate and lipid metabolism. Subsequent studies have also highlighted specific gene regulatory effects of $n-3$ and $n-6$ PUFA in tissues other than the liver (see pp. 352-353).

\section{Regulation of gene transcription}

Down-regulation of gene expression in liver and hepatocytes

A decrease in the synthesis of a particular protein, as opposed to a decrease in activity alone, implies a decrease in the transcription abundance of that specific protein's mRNA at the gene level, a decrease in the stability of the transcribed mRNA or a decreased translational capability.

A number of studies have now clearly shown that PUFArich diets result in the reduction of hepatic abundance of a variety of enzyme proteins, including FAS (Clarke et al. 1990), acetyl-CoA carboxylase (Salati \& Clarke, 1986), glucokinase (Jump \& Clarke, 1999), L-pyruvate kinase (Clarke et al. 1990), glucose-6-phosphate dehydrogenase (Jump \& Clarke, 1999), ATP-citrate lyase, malic enzyme, SCD1 (Ntambi, 1992; Landschulz et al. 1994; Sessler \& Ntambi, 1998; Jump \& Clarke, 1999), $\Delta^{5}$-desaturase (Cho et al. 1999a), $\Delta^{6}$-desaturase (Cho et al. 1999b), A-I, S14 
protein (Clarke et al. 1990) and glucose transporter-4 (Tebbey et al. 1994) involved in glucose metabolism, lipid synthesis and further metabolism and transport of fatty acids (Clarke, 2001). These studies also showed that the reduction in enzyme protein abundance elicited by PUFA occurred in the lipid synthetic or anabolic pathways and was largely due to a reduction in hepatic mRNA formation for the enzymes at the gene transcription level (Clarke, 2001). Many of the negative effects on gene transcription are elicited by both $n-3$ and $n-6$ PUFA, as is the case for the reduction in FAS and S14 mRNA. These effects were specific because phosphoenolpyruvate carboxykinase (PEPCK), a glucogenic enzyme, and actin, a structural protein, were not affected (Jump \& Clarke, 1999; Clarke, 2001). Whether these effects on liver enzyme transcription are due to the direct action of PUFA or to some of their metabolites is still debatable. The fact that specific prostanoid inhibitors did not reduce the inhibitory effect of PUFA on gene expression again suggested that PG are not necessary for the PUFA effects on hepatic fatty acid-synthesising enzymes to be manifested (Jump et al. 1993; Duplus et al. 2000). This is not the case for all enzymes and proteins in different tissues (see p. 352).

\section{Down-regulation of gene expression in adipose tissue and adipocytes}

Much of the early work on PUFA regulation of gene expression related to liver tissues and cells. However, the PUFA responses of specific enzymes and metabolicallyimportant proteins are not liver specific and have also been observed in other tissues, including adipose tissue and adipocytes (Sessler \& Ntambi, 1998; Duplus et al. 2000). PUFA effects on adipose tissue gene expression (FAS, lipoprotein lipase) appear to be site-specific. In the rat they occur retroperitoneal (internal) but not in subcutaneous inguinal (external) white adipose tissue and the effect appears to be specific for $n-3$ PUFA (Raclot et al. 1997). These differences in response of different adipose tissue sites may be a reflection of the known variation in metabolism between these sites (Sessler \& Ntambi, 1998). Suppression of the expression of lipogenic genes in 3T3-L1 adipocytes by ARA occurred by a mechanism that was dependent on PG formation (Jump \& Clarke, 1999). This finding was in sharp contrast to the reported lack of eicosanoid involvement in the suppression of the liver enzymes (see p. 351). A high- $n-6$ PUFA diet, when fed to obese $(f a / f a)$ and lean $(F a / F a)$ Zucker rats, suppressed the mRNA for SCD1 in adipose tissue of both phenotypes by about $75 \%$ relative to control diets. SCD1 mRNA in obese rats was much greater than that in lean animals (Jones et al. 1996). This finding supports the earlier findings of Wahle \& Radcliffe (1977), who suggested that the geneticallydetermined overexpression of SCD1 could still be inhibited by dietary LA. In cell culture systems, using the 3T3-L1 adipocyte cell line, Sessler et al. (1996) observed that $n-6$ PUFA (ARA, LA) and $n-3$ PUFA (EPA) were equally effective in reducing SCD1 mRNA in a dose-dependent manner, and that the effect was due primarily to a decrease in the stability of the mRNA. It is not clear if adipocytes from different body sites would also exhibit the differential regulatory effects of $n-6$ and $n-3$ PUFA reported in whole adipose tissue (Raclot et al. 1997).

Leptin is a hormone derived from adipose tissue and adipocytes that raises energy expenditure through activation of specific receptors in the hypothalamus (Reseland et al. $2001 b$ ). Its expression and plasma concentration correlates closely with adipose tissue mass in animals and man (Considine, 1997; Reseland et al. 2001b). High plasma leptin levels have been reported for volunteers on a high-fat diet compared with a low-fat diet, indicating that dietary fat also has a regulatory function. This role was further indicated when long-term decreased saturated fat intake and increased polyunsaturated fat intake attenuated plasma leptin concentrations in human volunteers beyond that expected on the basis of the change in body fat mass (Reseland et al. 2001a). Dietary $n-3$ PUFA also reduced plasma leptin concentrations in rats and resulted in lower leptin mRNA abundance in rat epididymal adipose tissue (Reseland et al. 2001b). In human trophoblasts (BeWo) in culture $n-3$ PUFA (both EPA and docosahexaenoic acid (DHA; $22: 6 n-3)$ ), albeit at high concentrations of $0 \cdot 5-1 \mathrm{mM}$, reduced leptin expression by about $70-80 \%$ compared with the saturated fatty acid control. In BeWo cells transfected with the human leptin promoter $n$-3 PUFA, but not monounsaturated or saturated fatty acids, reduced promoter activity (Reseland et al. 2001a). This finding correlated with decreased transcription factor mRNA content (peroxisome proliferator-activated receptor $\gamma$ and sterol regulatory element-binding protein (SREBP)-1). Possible mechanisms for the effects of PUFA on gene expression of various enzymes, cytokines and hormones are discussed in more detail (see pp. 354-355).

\section{Up-regulation of gene expression in liver and hepatocytes}

Fatty acids, both saturated fatty acids and PUFA, also exert positive regulatory effects on a number of hepatic enzymes involved in their transport, storage or disposal. These effects suggest that the mechanisms differ from those underlying negative regulation where only PUFA were effective (see p. 351). The up-regulated enzymes include acyl-CoA oxidase, acyl-CoA synthetase, carnitine palmitoyltransferase 1, liver fatty acid-binding protein, cytochrome P450 A1, hydroxymethylglutaryl-CoA synthase and cholesterol 7- $\alpha$ hydroxylase (Duplus et al. 2000). Upregulation by saturated fatty acids and PUFA requires protein synthesis de novo, since it is prevented by cycloheximide. Also, recent studies show that inhibitors of fatty acid oxidation and eicosanoid synthesis do not prevent up-regulation of carnitine palmitoyltransferase 1, thereby precluding these fatty acid derivatives as the actual regulators or secondary messengers (Duplus et al. 2000).

\section{Up-regulation of gene expression in adipose tissue and adipocytes}

A number of adipocyte genes are induced at the transcriptional level (mRNA) by treatment with fatty acids. These genes include the lipid-binding protein (adipocyte fatty acid-binding protein), acyl-CoA synthetase, fatty acyl-CoA transferase, lipoprotein lipase, glucose transporter-4, 
PEPCK and uncoupling protein 2 (Sessler \& Ntambi, 1998; Duplus et al. 2000). These enzymes are involved in adipogenesis, and fat storage in adipocytes and their induction probably reflects the potent regulatory effect of fatty acids on adipose differentiation. The observation that the induction of many of these enzymes is rather slow and can be inhibited by cycloheximide (e.g. adipocyte fatty acid-binding protein) indicates that protein synthesis is necessary for full expression and that fatty acids may not act directly on the gene but through a secondary signal mechanism such as a transcription factor (see p. 355; (Sessler \& Ntambi, 1998; Duplus et al. 2000; Clarke, 2001).

Clearly, both the positive and negative regulation of enzymes observed in specific metabolic pathways by fatty acids is not haphazard and non-specific, but is tightly coordinated and physiologically relevant to the metabolic requirements of the particular tissue under specific dietary conditions (lipid excess or deficiency). Increased availability of fatty acids from the diet down regulates the enzymic apparatus required for their synthesis de novo but up regulates that necessary for their oxidation, further metabolism and storage.

This coordinated regulation of gene expression in different tissues is exemplified by the fatty acid regulation of PEPCK expression in liver and adipose tissue and adipocytes (Duplus et al. 2000). In adipocytes monounsaturated fatty acids and PUFA, but not saturated fatty acids, markedly induce the content of PEPCK mRNA. The effect is rapid and does not require protein synthesis, suggesting that the fatty acids act directly on transcription of the PEPCK gene (Forest et al. 1997). Furthermore, inhibition of eicosanoid synthesis or increasing antioxidant supply had no effect on the enzyme induction, indicating that lipid-derived second messengers were not involved (Duplus et al. 2000). PEPCK activation in adipocytes by fatty acids increases the supply of glycerophosphate and consequently fatty acid esterification and subsequent storage. The fact that fatty acids have no effect on PEPCK regulation in liver, where it functions as a gluconeogenic enzyme, emphasises the physiological relevance of coordinated regulation by fatty acids, since glucose inhibits fatty acid stimulation of the enzyme in adipocytes (Duplus et al. 2000).

\section{Regulation of gene expression by fatty acids in other tissues and cells}

The regulation of enzyme gene expression by fatty acids is not restricted to carbohydrate and lipid-metabolising enzymes in liver and adipose tissue, but has been observed in a variety of other tissues and cells.

Immune tissue regulation. PUFA elicit a number of functional changes in immune cells, particularly proliferation, differentiation, necrosis and apoptosis. ARA and EPA inhibit proliferation of HL-60 leukaemia cells (Finstad et al. 1994), and various PUFA, including conjugated linoleic acids (CLA), inhibit proliferation of blood monocytes in culture (Cook et al. 1999). A number of studies have reported the attenuation of inflammatory cytokine formation by long-chain $n$-3 PUFA, particularly EPA and DHA, both in vivo-ex vivo and in cells in culture (Meydani et al. 1991; Robinson et al. 1995; Rotondo, 1995; Calder, 1997;
Wahle \& Rotondo, 1999). Furthermore, the inhibition of inflammatory cytokine production has been ascribed to a reduction in cytokine gene expression (transcription and translation) as determined by the lower abundance of mRNA and specific protein. Robinson et al. (1995) fed mice diets containing $n-3$ PUFA or beef tallow for 3-12 weeks. They isolated peripheral blood mononuclear cells and stimulated them ex vivo with lipopolysaccharide $(0 \cdot 1-10 \mathrm{ng} / \mathrm{ml})$ or phorbol myristate acetate $(8 \mathrm{pM}-8 \mu \mathrm{M})$. Northern analysis showed that cells from the $n-3$ PUFA-fed animals produced lower levels of interleukin $1 \beta$ mRNA, but with similar stability, in response to stimulation than the beef tallow-fed controls. Unfortunately, the efficacy of $n-3 v \cdot n$-6 PUFA was not determined by these authors.

The effect of $n-3$ PUFA on cytokine production can differ between individuals, and a small proportion of the population appear to have a genetic polymorphism whereby $n$-3 PUFA tends to increase inflammatory cytokine formation, in direct contrast to the general responses observed (Grimble et al. 2002). It is conceivable that the cytokine gene expression is up regulated by $n-3$ PUFA in these individuals, but this effect has not been reported. Clearly, a small proportion of the population may not derive anti-inflammatory benefit from $n$-3 PUFA and could be at risk of exacerbating any inflammation and its consequences. In view of the role of inflammation in cardiovascular disease, these individuals need to be identified and given specialist dietary advice.

Both $n-3$ and $n$-6 PUFA reportedly induce the expression of Thy-antigen on a T-lymphocyte cell line (Deglon et al. 1995). In a lymphoma cell line ARA has also been shown to decrease the expression of SCD2 (Tebbey \& Buttke, 1993).

CLA have also been reported to inhibit the inflammatory cytokine responses in immune tissues and enhance the immunoglobulin responses in a similar manner to that observed for $n$-3 PUFA in animals and man (Pariza, 1999; Sugano et al. 1999; I Mohede, R Albers, R van der Wielen, L Brink and V Dorovska-Taran, unpublished results).

Kidney. Inflammatory cytokine formation is also attenuated in autoimmune lupus nephritis-prone mice by diets supplemented with menhaden fish oil ( $n$-3 PUFA; Chandrasekar \& Fernandes, 1994). These authors showed by Northern analysis and immunoblotting that renal interleukin $1 \beta$ and 6 and tumour necrosis factor $\alpha$ mRNA and protein levels were completely suppressed by the $n$-3 PUFA, but were present in abundance in maize oil-fed animals. The fish oil diets delayed the onset of kidney disease and prolonged the lifespan of the animals compared with those fed maize oil. The authors also observed an increase in the expression of glutathione peroxidase, catalase and superoxide dismutase mRNA, enzymes that enhance the cell's ability to combat oxygen-derived free radical damage. These observations emphasised the opposing effects that can often be elicited by $n-6$ and $n-3$ PUFA on inflammation, and their differing effects on the regulation of the genes involved in the process. They also indicated that feeding maize oil could enhance the inflammatory process through pro-inflammatory cytokine and eicosanoid production. These authors reported previously that kidneys from maize oil-fed mice expressed higher levels of transforming growth factor $\beta 1$, intercellular adhesion molecule-1 and 
fibronectin-1 mRNA and protein, which emphasised the pro-inflammatory nature of maize oil and $n-6$ PUFA and their ability to regulate expression of specific genes, either directly or through some oxidative signal mechanism (Chandrasekar et al. 1995). These findings have relevance to diseases other than those of the kidney (see p. 352).

Cardiovascular tissue and cells. Cardiovascular disease is rapidly being recognised as a disease with a major immune-inflammatory component in its aetiology (Witztum \& Steinberg, 2001) and both $n-3$ PUFA and CLA have been implicated as preventative or ameliorating dietary factors (Meydani et al. 1991; Calder, 1997; Farquharson et al. 1999; Wahle \& Rotondo, 1999). Various stimuli, including oxidative stress, oxidised lipids, haemodynamic flow and inflammatory cytokines can up regulate the expression of a number of genes in the endothelium and circulating blood mononuclear cells that are responsible for the response of the tissue to stress. These genes include those encoding for adhesion molecules, inflammatory cytokines, heat-shock proteins, eicosanoids and redox enzymes (Yaqoob, 1998; Wahle \& Rotondo, 1999; Witztum \& Steinberg, 2001). Up-regulation of adhesion molecules, particularly intercellular adhesion molecule-1, vascular cell adhesion molecule and both E- and P-selectin and their co-ligands VLA-4 and LFA-1 (both members of the integrin family of transmembrane proteins) on endothelium and circulating mononuclear cells respectively, is important in recruiting these immune cells for normal immune functions. However, excessive expression of these molecules and a concomitant oxidative modification of LDL, which enhances their uptake by the scavenger receptors on resident macrophages in the intima of the vessel wall, constitute a primary event in the aetiology of atherosclerotic plaque formation and are implicated in the initiation of cancer metastases. Circulating cancer cells are also recruited to tissue sites by this process (Yaqoob, 1998; Wahle \& Rotondo, 1999; Witztum \& Steinberg, 2001). As mentioned earlier, n-3 PUFA attenuate the gene expression of inflammatory cytokines. A small number of laboratories, including ours, have reported that $n-3$ PUFA also decrease the enhanced expression (mRNA and protein) of cytokine-induced adhesion molecules (intercellular adhesion molecule-1, vascular cell adhesion molecule, E-selectin) in endothelial cells when compared with n-6 PUFA. (De Caterina et al. 1994; Collie-Duguid \& Wahle, 1996; De Caterina \& Libby, 1996; Collie-Duguid, 1997) and blood mononuclear cells (Hughes et al. 1996; Hughes \& Pinder, 1997). DHA was more effective than EPA in primary human umbilical vein endothelial cells and the addition of quercetin, a polyphenolic antioxidant, further decreased adhesion molecule expression (Collie-Duguid, 1997). The latter finding suggested that antioxidant status might play a role in adhesion molecule regulation. Recent observations indicated that CLA, as a mixture of the two main isomers (cis-9, trans-11 and trans-10, cis-12), also attenuated adhesion molecule expression at the mRNA level in human umbilical vein endothelial cells, similar to that observed for $n$-3 PUFA, and also at the protein level in vascular smooth muscle cells (Farquharson et al. 1999; M Goua, D Rotondo and KWJ Wahle, unpublished results), Dietary CLA have been reported to reduce atherosclerosis in rabbits on a high-cholesterol atherogenic diet and even to elicit a regression of plaque formation (Kritchevsky, 1999; D Kritchevsky, unpublished results). This outcome may be partly explained by their effects on adhesion molecule expression in vascular tissue (Farquharson et al. 1999). CLA also reduce inflammatory cytokine and eicosanoids, again similar to $n$-3 PUFA, and like $n-3$ PUFA are doubly effective as anti-atherogenic factors in being able to reduce both the inflammatory stimulus (cytokines; Cook et al. 1999; Sugano et al. 1999) and the response to that stimulus (adhesion molecules) through inhibition of specific gene expressions (Farquharson et al. 1999).

Both $n$-3 PUFA (EPA and DHA) and CLA up regulate the expression of the redox enzymes (mRNA and activity) glutathione peroxidase 1 , glutathione $S$-transferase and phospholipid hydroperoxide glutathione peroxidase 4 in primary human umbilical vein endothelial cells (Crosby et al. 1996; Wahle et al. 1997; Wu et al. 1998). These findings are similar to those observed in autoimmunediseased kidneys (see p. 352). This outcome could be a response to oxidative stress elicited by the PUFA and may represent an important mechanism for regulating gene expression in the stress response pathways (see p. 352).

Gastrointestinal tissue and cells. PUFA also regulate the expression of genes in the gastrointestinal tract, including L-fatty acid-binding protein, apolipoprotein A-IV and apolipoprotein $\mathrm{C}$-III in gastric tissue and acetyl-CoA carboxylase, leptin and prolactin in pancreatic $\beta$ cells (Niot et al. 1997; see also Sessler \& Ntambi, 1998; Briscoe et al. 2001). It is not clear whether these outcomes are due to direct effects of PUFA or indirect effects through various signal and transcription mechanisms (see pp. 354-358).

Cancer tissue and cells. Dietary fat has been implicated in the aetiology of many forms of cancer but, as observed for atherogenesis, not all fats are regarded as pro-carcinogenic. Both $n$-3 PUFA (particularly EPA and DHA) and CLA appear to exhibit anti-carcinogenic and anti-metastatic properties in animals and to inhibit cancer cell growth in vitro, whereas the parent fatty acid of CLA, LA, has the opposite effect. LA has been shown to increase tumour growth and development in rodent models of various cancers and to enhance tumour cell proliferation (Wahle \& Heys, 2002). Ip et al. (1995, 1996, 2001) have shown that feeding female rodents on CLA until puberty reduced their susceptibility to induction of mammary tumours by chemical agents in post-pubertal life. This finding suggested that CLA positively affected certain aspect(s) of mammary differentiation and development that prevented or ameliorated cancer incidence throughout life. These observations would be of major importance in the prevention of breast cancer if they also pertained to prepubertal girls. Ip et al. (2001) were also the first to suggest that the anti-carcinogenic effects of CLA were due to an induction of apoptosis in rodent breast tumours. They observed reduced protein expression of the antiapoptotic proto-oncogene bcl-2, using immunohistochemical analysis, in mammary tissue of rats fed CLA in comparison with control diets. In contrast, neither apoptosis nor the apoptotic regulatory genes were affected by CLA in 
normal mammary alveoli or terminal end buds. Changes in the expression of other oncogenes at the mRNA or protein level were either not detected (e.g. bak and bax) or not investigated (e.g. pro-apoptotic p53, p21WAF1/CIP1, bad, bcl-Xs).

It has been shown that CLA, but not LA, inhibit cancer cell proliferation, induce DNA damage and regulate the gene expression of opposing pro- and anti-apoptotic oncogenes in breast and prostate cancer cells in a specific and coordinated manner (Farquharson et al. 1999; Majumder et al. 2002; JJH Ochoa, B Majumder, SD Heys, A Farquharson, A Schofield and KWJ Wahle, unpublished results). CLA attenuated the expression of the anti-apoptotic cell-protective bcl-2 at the level of mRNA and protein in human prostate cancer and breast cancer cells and induced the expression of pro-apoptotic p53 and p21WAF1/CIP1. Breast cancer cells appeared more sensitive to CLA than prostate cancer cells and the trans-10, cis-12 isomer was more effective than the cis-9, trans-11 isomer in the prostate cells. Mutation of the p53 gene, as observed in the breast cancer cell line MBA-MD-231, expresses a mutant protein that prevents apoptosis and enhances carcinogenesis. It has been observed that CLA could suppress the expression of this mutant p53 protein; the first indication that diet might affect the expression and action of mutagenic products (Majumder et al. 2002).

Other tissues. PUFA regulation of the expression of specific genes at the mRNA and protein level in a variety of other tissues have been reported, including the Na channel in cardiomyocytes and SCD2 in the brain (Sessler \& Ntambi, 1998). The question of how PUFA elicit their effects on gene transcription is intriguing and remains to be clarified (see pp. 354-355).

\section{Cellular mechanisms involved in polyunsaturated fatty acid regulation of gene transcription}

Precisely how PUFA and their various metabolic derivatives elicit their specific and coordinated regulatory effects on the expression of such a wide variety of genes involved in such a multitude of complex metabolic pathways is still being debated and is regarded as a 'hot topic' of research. What is clear is that dietary PUFA are able to regulate cellular signal cascades, both positively and negatively, and ultimately influence the 'control centre' of the cell, either directly or indirectly, to alter its production of regulatory proteins. These gene regulatory effects of PUFA have profound effects on cell and tissue metabolism and offer a credible explanation for the involvement of nutrient-gene interactions in the initiation and prevention or amelioration of diseases such as obesity, diabetes, cardiovascular disorders, immune-inflammatory diseases and cancers. Understanding the molecular mechanisms that can be regulated by PUFA could also result in novel pharmaceutical products and more effective interventions and therapies for common diseases.

PUFA and their derivatives have been postulated to exert their effects on gene expression at different points in the signal cascades, including direct interaction with fatty acid response elements in the promoter regions of certain genes (Jump \& Clarke, 1999; Duplus et al. 2000; Clarke, 2001). These generalised mechanisms, for which there is supporting evidence in the literature, are depicted in Fig. 1 (adapted from Duplus et al. 2000; Clarke, 2001). They are:

A. dietary PUFA, PUFA-CoA and PUFA metabolites such as eicosanoids and oxidation products (hydroxyeicosatetraenoates, hydroperoxyeicosatetraenoates, hydroxyoctadecenoates) can activate cell

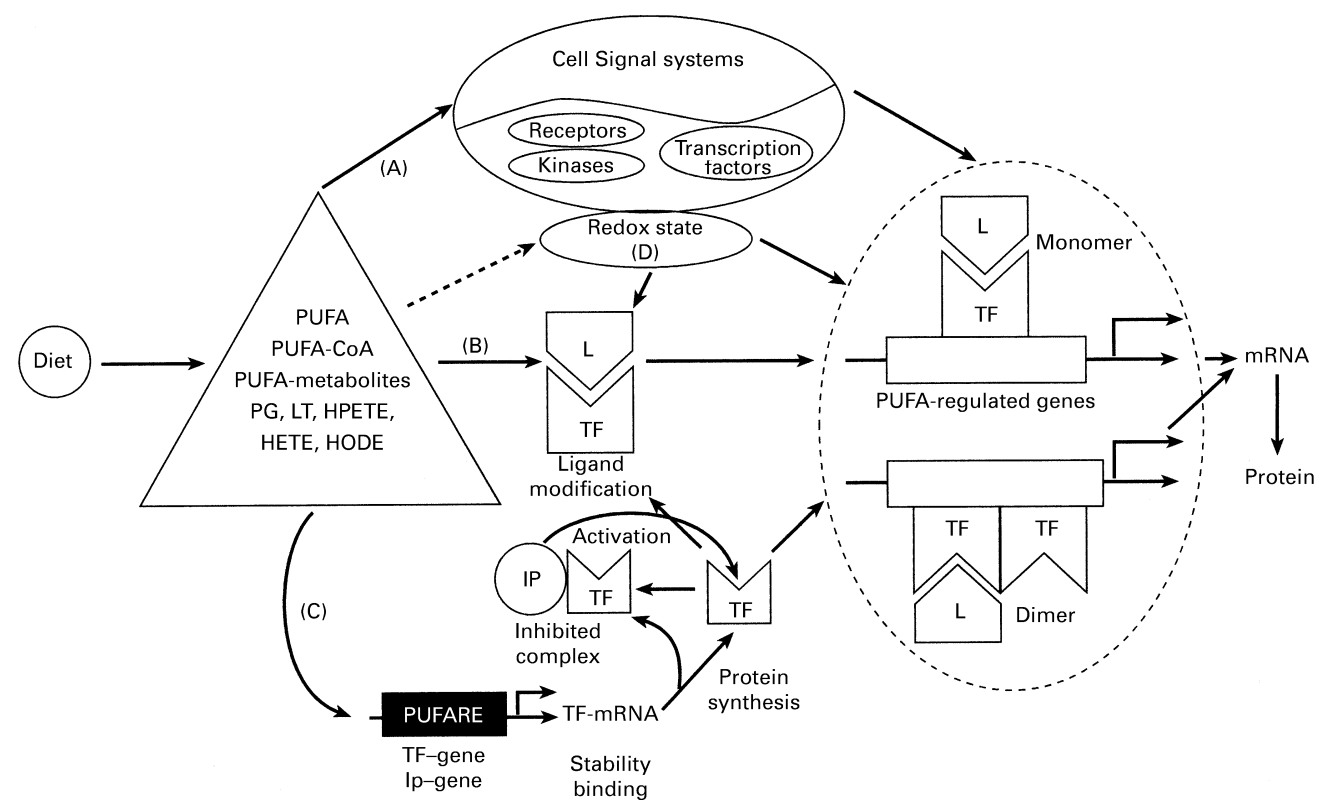

Fig. 1. Possible mechanisms of polyunsaturated fatty acids (PUFA)-regulated gene expression. For explanations of possible mechanisms (A-D), see, pp. 354-355. PUFARE, polyunsaturated fatty acid-response element; TF, transcription factor; L, ligand; PG, prostaglandins; LT, leukotrienes; HPETE, hydroperoxyeicosatetraenoates; HETE, hydroxyeicosatetraenoates; HODE, hydroxyoctadecenoates; Ip, inhibitory protein. (Adapted from Duplus et al. 2000 and Clarke, 2001.) 
signal cascades (receptors, kinases). This action in turn can result in the covalent modification (e.g. phosphorylation) of specific transcription factors resulting in their activation, nuclear translocation and binding to specific DNA recognition sequences, or PUFA-response elements, in the promoter region of target genes either as monomers, homodimers or heterodimers. Transcription factor binding to the specific promoters results in up- or down-regulation of the transcription (mRNA) of the specific proteins and the proteins per se;

B. the fatty acid or its derivatives can act as ligands and bind to specific transcription factors, which enhances their capacity to bind to recognised fatty acid-response elements in the promoter regions of target genes, again in monomeric or various dimeric forms. The end point is again activation or suppression of transcription;

C. PUFA and PUFA-derivatives are also able to modify transcription of transcription factor mRNA per se and possibly that of an inhibitor protein by direct or indirect mechanisms. They may also alter the stability of a particular transcription factor mRNA and/or its DNA-binding capacity. These effects would also result in modulation of gene expression;

D. PUFA and their derivatives may utilise a number of indirect mechanisms to regulate gene expression. Of particular interest in this regard is their known modulation of the intrinsic redox state of the cell, which in turn can regulate transcription factor activation and nuclear binding (e.g. nuclear factor- $\kappa \mathrm{B}(\mathrm{NF}-\kappa \mathrm{B})$ and its

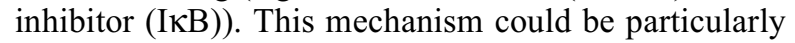
important in regulation of the stress-response genes.

\section{Transcription factors}

\section{Peroxisome proliferator-activated receptors}

The nuclear hormone receptors comprise a family of transcription factors involved in regulation of differentiation and cell and tissue homeostasis, particularly lipid homeostasis. Their transcriptional activity is tightly controlled by a variety of lipophilic ligands. Peroxisome proliferator-activated receptors (PPAR) are orphan nuclear receptors of this family that are activated by micromolar concentrations of fatty acids and their derivatives and analogues including the hypolipidaemic fibrate drugs, peroxisome proliferators, PUFA and oxidised PUFA derivatives including specific PG, hydroxyeicosatetraenoates, hydroxyoctadecenoates and leukotrienes (Jump \& Clarke, 1999; Duplus et al. 2000; Kersten et al. 2000; Clarke, 2001; Chawla et al. 2001).

Three isoforms of PPAR have been cloned, PPAR- $\alpha,-\delta$ and $-\gamma$, that show tissue-specific expression, specific ligandbinding preferences and a common ability to form heterodimers with the retinoid $X$ receptors $\alpha, \beta$ and $\gamma$. It is the PPAR-retinoid $X$ receptor complex that is the active transcription factor that binds the DNA sequence elements termed peroxisome proliferator response elements in the promoter regions of target genes (Jump \& Clarke, 1999; Duplus et al. 2000; Kersten et al. 2000; Clarke, 2001; Chawla et al. 2001). Peroxisome proliferator response elements have been identified in a number of genes involved in lipid homeostasis and cell differentiation and regulation.
They comprise imperfect AGGTCA consensus sequences separated by a one-nucleotide direct repeat with an additional 5'-extended AACT portion (Duplus et al. 2000; Chawla et al. 2001). Using transactivation assays, where cells are transfected with a PPAR expression vector and a reporter gene controlled by a promoter containing a response element that is activated by the PPAR, it was shown that PUFA and its derivatives are PPAR activators (Kliewer et al. 1997; Duplus et al. 2000; Kersten et al. 2000; Clarke, 2001). Evidence that PUFA and their derivatives are true ligands for PPAR was obtained using competitive binding assays with labelled known ligands. Certain monounsaturated fatty acids and PUFA were shown to bind directly to both PPAR- $\alpha$ and PPAR- $\gamma$ and the lipoxygenasederived eicosanoid $8(\mathrm{~S})$-hydroxyeicosatetraenoic acid and cyclooxygenase-derived 15 -deoxy- $\Delta^{12,14}-\mathrm{PGJ}_{2}$, a cyclopentenone PG, derived from ARA, were shown to be potent selective activation ligands for PPAR- $\alpha$ and PPAR- $\gamma$ respectively. Leukotriene $\mathrm{B}_{4}$ derived by the action of 5-lipoxygenase is also a specific PPAR- $\alpha$ ligand. Furthermore, carbaprostacyclin, a stable analogue of $\mathrm{PGI}_{2}$ is known to activate both PPAR- $\alpha$ and PPAR- $\delta$ (Kliewer et al. 1997). Clearly, cyclooxygenase and lipoxygenase products of PUFA oxidation can elicit differential PPAR activations, assuming that they are formed in the specific cells that express the different isoforms.

PPAR- $\alpha$ appears to be mainly localised in hepatic tissues and cells. The PPAR- $\gamma_{2}$ isoform is selectively expressed in adipose tissue and adipocytes, but not in pre-adipocytes, and when transfected into non-adipocytes it induces their differentiation to adipocytes. However, PPAR- $\gamma$ is also expressed in other tissues and cells, particularly those involved in immune functions such as intestinal mucosa and blood mononuclear cells where they are implicated in the suppression of inflammatory cytokine formation and inducible NO synthetase (iNOS; Davidson \& Rotondo, 2000; Rotondo \& Davidson, 2002). A recent study clearly showed that supplementation of pigs with CLA before induction of enteric inflammation with orally-administered bacteria completely inhibited mucosal inflammation and lesion formation. The inhibition of the inflammatory cytokine profile correlated with enhanced expression or activation of PPAR- $\gamma$ in the tissue, indicating that the actions of CLA are PPAR- $\gamma$-mediated. This suggestion was supported by studies using the RAW264.7 mouse macrophage cell line in which CLA isomers were shown to activate PPAR- $\gamma$ and simultaneously decrease the interferon$\gamma$-induced expression of $\mathrm{mRNA}$ and protein of inflammatory mediators such as cyclooxygenase-2, iNOS, tumour necrosis factor $\alpha$ and interleukin-1 $\beta$ (Yu et al. 2002). The requirement for a functional PPAR- $\gamma$ protein in the CLA-induced suppression of iNOS in RAW264.7 cells was emphasised by the abolition of the CLA effect when cells were transfected with a dominant negative PPAR- $\gamma$ mutant construct.

CLA are also potent direct PPAR- $\alpha$ activators, as evidenced by the fact that they were able to displace the labelled PPAR- $\alpha$-specific synthetic ligand GW2331 at low concentrations $(140 \mathrm{nM})$. CLA were also able to activate PPAR- $\alpha$ fivefold at concentrations as low as $5 \mu \mathrm{M}$ (Moya-Camarena et al. 1999). 
Clearly, CLA, other PUFA and their derivatives are extremely potent activators of PPAR, and through this activation are able to regulate the expression of proinflammatory cytokines, cyclooxygenase and lipoxygenase derivatives and iNOS. The possibility that different concentrations of CLA may activate different PPAR (i.e. low concentrations activate PPAR- $\alpha$ and higher concentrations activate PPAR- $\gamma$ ) warrants further investigation.

It is conceivable that the multiple beneficial effects on health attributed to CLA could relate to their effects on the activation of these important transcription factors (Wahle \& Rotondo, 1999).

Although there is evidence that PPAR play a major role in the PUFA regulation of gene expression through a PPAR-retinoid $\mathrm{X}$ receptor-peroxisome proliferator response element-mediated process (see Fig. 1), recent evidence suggests that modulation of gene expression by PUFA and other PPAR activators is not always linked. For example, the genes encoding apolipoprotein A-II in hepatocytes and fatty acyl-CoA transferase-CD36 in differentiated adipocytes contain a peroxisome proliferator response element in their promoter regions, but they are not regulated by fatty acids (Duplus et al. 2000). Similarly, the genes encoding $\Delta^{5}$ - and $\Delta^{6}$-desaturases are suppressed by PUFA but up regulated by PPAR activators (Cho et al. $1999 a, b)$. In hepatocytes carnitine palmitoyltransferase 1 gene expression induced by fibrates is attenuated when lipoxygenases are inhibited, but fatty acids are still effective modulators, and PUFA can modulate carnitine palmitoyltransferase 2 gene expression whereas peroxisome proliferators are ineffective (Duplus et al. 2000). A divergence in PUFA effects and PPAR effects was also observed in PPAR- $\alpha$ knock-out mice, in which $n$-3 PUFA administration in vivo or to hepatocytes derived from these mice suppressed S14 and FAS gene expression linked to lipogenesis but did not induce the normally-expected catabolic lipid-disposal genes such as acyl-CoA oxidase and cytochrome P450 A2 (Ntambi, 1992; Sessler \& Ntambi, 1998; Duplus et al. 2000).

From the foregoing evidence it is clear that PPAR are important in the PUFA regulation of gene transcription, but that they are possibly not the only transcription factors through which PUFA can elicit their effects.

\section{Other fatty acid-responsive transcription factors}

\section{Sterol regulatory element-binding protein}

Genes involved in fatty acid, glucose and cholesterol metabolism contain sterol regulatory elements in their promoter regions. SREBP are a family of transcription factors (SREBP-1a, -1c and -2) first identified as ligands for sterol regulatory elements that are post-transcriptionally regulated. SREBP-2 regulates genes involved in cholesterol metabolism, whilst SREBP-1a and -1c regulate genes involved in both lipogenesis and cholesterolgenesis (Jump \& Clarke, 1999; Clarke, 2001). SREBP-1 is synthesised as a $125 \mathrm{kDa}$ precursor protein attached to the endoplasmic reticulum. The active $68 \mathrm{kDa}$ protein is released by proteolytic cleavage in the Golgi system, which is facilitated by the SREBP cleavage-activating protein. Active SREBP-1 then translocates to the nucleus where it binds to sterol regulatory elements. Up-regulation of SREBP-1a expression in liver elicits high expression of fatty acid-synthesising enzymes and enhanced fatty acid biosynthesis, whereas down-regulation of this factor has the opposite effect (Jump \& Clarke, 1999; Duplus et al. 2000; Clarke, 2001). PUFA, particularly $n$-3 PUFA, suppress lipid synthesis initially, in a few minutes post- $n-3$ PUFA feeding, by rapidly inhibiting the proteolytic release of active SREBP-1 from its membrane-anchored precursor. This action reduces the upstream DNA binding of the co-transcription factors NF-Y and $\mathrm{Sp} 1$, which are activators of the insulin response regions in specific genes. Longer-term n-3 PUFA intake then inhibits SREBP-1 mRNA abundance in hepatic cells by accelerating its decay time. The consequence of this effect is a reduced amount of the SREBP-1 precursor in hepatic tissue. It has been postulated that PUFA may also inhibit the DNA binding of transcription factors such as hepatic nuclear factor 4, but this effect remains to be clarified (Clarke, 2001). The effects of PUFA on SREBP-1 are supported by observations in transgenic mice that either overexpress or are a knockout for the gene (Duplus et al. 2000). Clearly, the effects of PUFA on SREBP-1 are both transcriptional (mRNA) and post-transcriptional, depending on the time over which the PUFA are present in the tissue or cell (Jump \& Clarke, 1999; Duplus et al. 2000; Clarke, 2001).

\section{Hepatic nuclear factor 4 and liver $X$ receptor}

Hepatic nuclear factor 4 does not bind fatty acids but is activated by fatty acyl-CoA derivatives. This role contrasts with the PPAR-retinoid X receptor system, which does not bind fatty acyl-CoA (Jump \& Clarke, 1999). Hepatic nuclear factor 4 binds to one-nucleotide direct repeat sequences in promoter regions of specific genes (apolipoprotein C-III, tyrosine amino transferase, PEPCK and L-pyruvate kinase) as a homodimer and elicits changes in gene transcription (Jump \& Clarke, 1999).

PUFA can also reduce the expression of liver $\mathrm{X}$ receptor, a nuclear receptor thought to be involved in the regulation of cholesterol, steroid hormone and bile acid catabolism. In this case and in the case of the c-fos and nur-77 expression PUFA appear to modulate the amount of the transcription factors, although it is not clear what the precise regulatory mechanism is at present (Duplus et al. 2000). PUFA have recently been shown to inhibit the expression of the SREBP-1c gene by antagonising the ligand-dependent activation of the liver $\mathrm{X}$ receptor (Ou et al. 2001).

\section{Nuclear factor $\kappa B$}

The transcription factor NF- $\mathrm{KB}$ is an inducible eukaryotic transcription factor of the rel family. It is a major component of the stress cascade that regulates the activation of early response genes involved in the expression of inflammatory cytokines, adhesion molecules, heat-shock proteins, cyclooxygenases, lipoxygenases and redox enzymes. In unstimulated cells NF- $\mathrm{KB}$ is present in the cytosol as an inactive precursor that is a heterodimer or homodimer of p50 and p65 and is complexed to its specific 
inhibitor protein I I B. Stimulation with various stress factors, including heat, oxidative stress and inflammatory cytokines, results in a specific I $\kappa \mathrm{B}$ kinase being activated that phosphorylates $\mathrm{IkB}$ on two serine residues. This activated form of the $\mathrm{I} \kappa \mathrm{B}-\mathrm{NF}-\kappa \mathrm{B}$ complex is then polyubiquitinated and undergoes proteosomal degradation, which releases free $\mathrm{NF}-\kappa \mathrm{B}$ and thereby facilitates its translocation to the nucleus. In the nucleus it binds specific $\kappa \mathrm{B}$ sequences in the promoter regions of the various genes encoding signal proteins and up regulates or down regulates their transcription activity (Brown et al. 1993; Huang et al. 2000; Haddad et al. 2002).

ARA, an $n-6$ PUFA, strongly up regulated NF- $\kappa$ B nuclear translocation in the U937 pro-monocytic cell line (Camandola et al. 1996), which suggested a stimulation of the previously described activation pathway to release $\mathrm{NF}-\kappa \mathrm{B}$ from its inhibitor I $\kappa \mathrm{B}$. This effect was not observed with EPA, an $n-3$ PUFA, which explains, at least in part, the respective pro- and anti-inflammatory effects of these PUFA in vivo (Meydani et al. 1991; Calder, 1997; Wahle \& Rotondo, 1999).

Up-regulation of NF- $\mathrm{NB}$ is also regarded as anti-apoptotic and could explain why $n-6$ PUFA enhance tumour growth in animal models (Mustapha et al. 2000; Natarajan et al. 2001). The inhibitory effects of inhibitors of eicosanoid synthesis, indomethacin and nordihydroguarinic acid, and the activation by $\mathrm{PGE}_{2}$ indicated that eicosanoid metabolites of ARA were responsible for the up-regulation of NF- $\mathrm{KB}$ activation (Camandola et al. 1996). This finding also suggested that EPA derivatives were either not effective or inhibitory. It is interesting, therefore, that cyclopentenone $\mathrm{PG}$ such as 15-deoxy-PGJ $\mathrm{J}_{2}$ and $\mathrm{PGA}_{2}$, which like $\mathrm{PGE}_{2}$ are also derived from ARA but via $\mathrm{PGD}_{2}$, actually inhibited multiple steps in the NF- $\mathrm{KB}$ cascade (Rossi et al. 1997; Straus et al. 2000). Furthermore, this PG is a potent activator of PPAR- $\gamma$ (see p. 355 and Straus et al. 2000). Clearly, the extent of ARA metabolism through different pathways can determine whether NF- $\kappa B$ is positively or negatively regulated, which increases the complexity of PUFA-gene regulations even further. The repression of a number of pro-inflammatory genes in activated macrophages by 15 -deoxy-PGJ $\mathrm{J}_{2}$, including iNOS and tumour necrosis factor $\alpha$, is at least partly due to activation of PPAR- $\gamma$ expression. Clearly, both PPAR and NF- $\kappa B$ have a role in regulating inflammatory responses.

The studies of Straus et al. (2000) in HeLa cells showed that 15 -deoxy-PGJ $\mathrm{J}_{2}$ potently inhibited the NF- $\mathrm{KB}$ activation after treatment with lipopolysaccharide by inhibiting the phosphorylation of two critical cysteine residues on IאB by $\mathrm{I} \kappa \mathrm{B}$ kinase. This activity prevents the degradation of the $\mathrm{NF}-\kappa \mathrm{B}-\mathrm{I} \kappa \mathrm{B}$ complex and the release of active NF- $\kappa \mathrm{B}$ for nuclear translocation and binding to target genes. The effects were kinase-specific in that protein kinase A was not affected and Jun-N terminal kinase was only slightly affected. 15-Deoxy-PGJ ${ }_{2}$ also abolished the DNA binding of highly-purified p65 and p50 NF- $\mathrm{BB}$ heterodimers in vitro (Straus et al. 2000). Similar inhibitory effects on phorbol ester-induced NF- $\kappa \mathrm{B}$ activation and nuclear translocation were observed with $\mathrm{PGA}_{1}$ (another cyclopentonene $\mathrm{PG}$ ) in Jurkat T cells, HeLa cells and lymphoid cells, as well as in human monocytes from healthy donors (Rossi et al. 1997). This finding highlighted the general nature of the effects of cyclopentenone PG in different cell types. Also, all the cyclopentenone $\mathrm{PG}\left(\mathrm{PGA}_{1}, \mathrm{PGA}_{2}\right.$, dimethyl $\mathrm{PGA}_{2}$, $\mathrm{PGJ}_{2}$, 15-deoxy- $\mathrm{PGJ}_{2}$ ) were effective, whilst non-reactive cyclopentenone $\mathrm{PGB}_{1}$ and non-cyclopentenone $\mathrm{PG}\left(\mathrm{PGE}_{1}\right.$, $\mathrm{PGD}_{2}, \mathrm{PGF}_{2} \alpha$ ) were ineffective in suppressing phorbol acetate-induced NF- $\kappa \mathrm{B}$ activation (Rossi et al. 1997). $\mathrm{PGA}_{1}$ treatment effectively inhibited the phorbol acetate-induced expression of reporter genes controlled by $\kappa \mathrm{B}$ sites in their promoter regions, but enhanced the expression of reporter genes with the heat-shock protein-70 promoter. From this finding it is evident that NF- $\mathrm{BB}$ up-regulation suppresses heat-shock protein-70 expression. Heat-shock transcription factor is up regulated within $60 \mathrm{~min}$ of $\mathrm{PGA}_{1}$ addition to levels observed after heat-shock stimulation and required new protein synthesis. This finding indicated an indirect regulation of heat-shock transcription factor by the cyclopentenone PG (Amici et al. 1992). Cyclopentenone PG have been reported to block HIV-1 mRNA transcription and this outcome could be due to their inhibitory effects on NF- $\kappa$ B activation (Rozera et al. 1996).

Cyclopentenone PG (15-deoxy-PGJ 2 ) also potently inhibited iNOS induction in lipopolysaccharide-activated micro-glial cells. This PG suppressed iNOS promoter activity, and both mRNA and protein levels of iNOS in the activated glial cells. Again this gene regulation did not involve the nuclear receptor-transcription factor PPAR- $\gamma$, since troglitazone, a known ligand for PPAR- $\gamma$, was not able to inhibit iNOS induction and neither 15-deoxy-PGJ $J_{2}$ nor troglitazone could activate a transfected PPAR-dependent promoter in the absence of a co-transfected PPAR- $\gamma$. In contrast to the findings of Straus et al. (2000) in HeLa cells (see p. 357), the 15-deoxy-PGJ $\mathrm{J}_{2}$ in glial cells did not block nuclear translocation or DNA binding of the NF- $\kappa$ B but did inhibit the activity of an NF- $\kappa \mathrm{B}$ reporter construct. This finding suggested that the mechanism of inhibition was most likely due to disruption of NF- $\mathrm{KB}$ transcription in the nucleus (Petrova et al. 1999).

It would appear that cyclopentenone $\mathrm{PG}$ inhibit various stages in the NF- $\kappa \mathrm{B}$ activation cascade. This inhibitory activity constitutes part of a product feedback mechanism resulting in the down-regulation of inflammation.

It has been shown that CLA can also regulate cytokineinduced NF- $\kappa \mathrm{B}$ activation in prostate cancer cells by attenuating the initial phosphorylation of $\mathrm{I} \kappa \mathrm{B}$ and reducing the nuclear translocation of NF- $\mathrm{KB}$ (Song et al. 2003; HJ Song, G Yu, CS Bestwick, AA Sneddon, S-N Choe, I Grant, SD Heys and $\mathrm{K}$ Wahle, unpublished results). Initially, in the short term $(6 \mathrm{~h}), \mathrm{CLA}$ activated $\mathrm{NF}-\kappa \mathrm{B}$ by increasing I $\mathrm{B}$ phosphorylation, but this effect was reversed on longer-term stimulation (24 h; Song et al. 2003; HJ Song, G Yu, CS Bestwick, AA Sneddon, S-N Choe, I Grant, SD Heys and K Wahle, unpublished results). It has been reported that activated NF- $\kappa \mathrm{B}$ also stimulates the synthesis of $\mathrm{I} \kappa \mathrm{B}$ mRNA, and newly-synthesised I $\mathrm{B}$ proteins are transported to the nucleus to bind and remove NF- $\kappa B$ from specific gene promoters. A nuclear export sequence on the I $\mathrm{B} B$ then facilitates the export of the NF- $\mathrm{B}$-I $\mathrm{I} \mathrm{B}$ complex to restore the pre-induction state (Huang et al. 2000). 


\section{Redox state, polyunsaturated fatty acids and gene transcription}

PUFA supplementation in animals and man in vivo and in cells in culture can induce the expression of genes encoding redox enzymes (catalase, glutathione peroxidase, superoxide dismutase), whilst suppressing the expression of genes encoding inflammatory cytokines and adhesion molecules (Chandresakar \& Fernandes, 1994; Crosby et al. 1996; Natarajan et al. 2001; Haddad et al. 2002). Similar effects on redox state and adhesion molecule expression have been observed with CLA, suggesting that these types of PUFA act through similar mechanisms (Wahle et al. 1997; Wu et al. 1998; Farquharson et al. 1999). NF-кB is known to be an important regulator of adhesion molecule and cytokine gene expression. Furthermore, both activation and nuclear DNA binding of NF- $\mathrm{KB}$ can be influenced by the redox state of the cells. It is conceivable, therefore, that the action of PUFA on gene transcription may be regulated through changes in cellular redox state in the first instant.

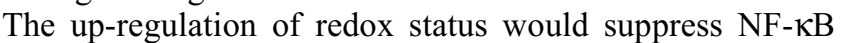
activation with a concomitant change in gene transcription and translation activity (both positive and negative depending on the genes, e.g. negative for cytokines, but positive for heat-shock protein-70). These observations suggest that regulation of stress-response genes may initially depend on the redox state of the cell, and that this state can be modified by PUFA, possibly through a transient oxidative stress that induces the redox enzymes.

\section{Conclusions}

From the foregoing it is clear that PUFA can regulate the expression of a number of genes, often in a coordinated and physiologically-relevant manner in which anabolic pathways are up regulated whilst catabolic pathways are down regulated, and vice versa. Regulation can be elicited by PUFA per se, by their acyl-CoA derivatives or by a variety of metabolic derivatives, including eicosanoids and other oxidative products. Regulation of gene expression by PUFA and their derivatives can also occur at a number of different points in the signal cascade, including effects on various kinases, transcription factors (ligands, nuclear binding and stability), redox enzymes, mRNA stability and post-transcriptional and translational effects. It is likely that with time many other derivatives of PUFA (in addition to those mentioned earlier) will be shown to have generegulatory properties, and that many more, as yet unknown, transcription factors will be identified as PUFA-regulated at the many possible sites described earlier. Clearly, PUFA regulation of gene expression is extremely complex, but very important if the way in which dietary fats can impact on health, both positively and negatively, is to be understood.

The present review is not meant to be all-encompassing, but seeks to emphasise the important role that PUFA play in gene regulation and cell physiology and metabolism, and to describe some of the complex interactive mechanisms involved. It further attempts to show how these PUFAregulated processes can impact on health. The readers are directed to the number of excellent reviews cited here that cover the subject in greater detail. Also, the concept of an overriding regulation by redox status requires further elucidation, but at present is intriguing.

\section{References}

Allmann DW \& Gibson DW (1969) Fatty acid synthesis during early linoleic acid deficiency in the mouse. Journal of Lipid Research 6, 51-62.

Amici C, Sistonen L, Santoro MG \& Morimoto RI (1992) Antiproliferative prostaglandins activate heat shock transcription factor. Proceedings of the National Academy of Sciences USA 89, 6227-6231.

Briscoe CP, Harit S, Arcj JR \& Tadayyon M (2001) Fatty acids inhibit leptin signalling in BRIN-BDII insulinoma cells. Journal of Molecular Endocrinology 26, 145-154.

Brown K, Park S, Kanno T, Franzoso G \& Siebenlist U (1993) Mutual regulation of the transcriptional activator NF- $\mathrm{\kappa B}$ and its inhibitor IKB- $\alpha$. Proceedings of the National Academy of Sciences USA 90, 2532-2536.

Calder PC (1997) N-3 polyunsaturated fatty acids and cytokine production in health and disease. Annals of Nutrition and Metabolism 41, 203-234.

Camandola S, Leonarduzzi G, Musso T, Varesio L, Carini R, Scavazza A, Chiarpotto E, Bauerle PA \& Poli G (1996) Nuclear factor $\kappa \mathrm{B}$ is activated by arachidonic acid but not by eicosapentaenoic acid. Biochemical and Biophysical Research Communications 229, 643-647.

Chandrasekar B \& Fernandes G (1994) Decreased proinflammatory cytokines and increased antioxidant enzyme gene expression by $\omega-3$ lipids in murine lupus nephritis. Biochemical and Biophysical Research Communications 200, 893-898.

Chandrasekar B, Troye DA, Venkatraman JT \& Fernandes G (1995) Diet, $\omega-3$ lipids delay onset and progress of autoimmune lupus nephritis by inhibiting TGF $\beta$ mRNA and protein expression. Journal of Autoimmunology 8, 381-393.

Chawla A, Repa JJ, Evans RM \& Mangelsdorf DJ (2001) Nuclear receptors and lipid physiology: opening the X-files. Science 294, 1866-1870.

Cho HP, Nakamura MT \& Clarke SD (1999a) Cloning, expression and nutritional regulation of the mammalian delta-6-desaturase. Journal of Biological Chemistry 274, 471-477.

Cho HP, Nakamura MT \& Clarke SD (1999b) Cloning, expression and fatty acid regulation of the human delta-5-desaturase. Journal of Biological Chemistry 274, 37335-37339.

Clarke SD (2001) Polyunsaturated fatty acid regulation of gene transcription: a molecular mechanism to improve the metabolic syndrome. Journal of Nutrition 131, 1129-1132.

Clarke SD, Armstrong MK \& Jump DB (1990) Dietary polyunsaturated fats uniquely suppress rat liver fatty acid synthase and S14 mRNA content. Journal of Nutrition 120, 225-231.

Collie-Duguid ESR (1997) Regulation of human endothelial ICAM-1, E-selectin and VCAM-1 by polyunsaturated fatty acids and anti-oxidants. PhD Thesis, University of Aberdeen.

Collie-Duguid ESR \& Wahle KWJ (1996) Inhibitory effect of fish oil n-3 polyunsaturated fatty acids on the expression of endothelial cell adhesion molecules. Biochemical and Biophysical Research Communications 220, 269-274.

Considine RV (1997) Weight regulation, leptin and growth hormone. Hormone Research 48, 116-121.

Cook ME, DeVoney D, Drake B, Pariza MW, Whigham L \& Yang M (1999) Dietary control of immune induced cachexia: CLA and immunity. In Advances in Conjugated Linoleic Acid Research, vol. 1, pp. 226-237 [MP Yurawecz, MM Mossoba, JKG Kramer, MW Pariza and GJ Nelson, editors]. Champaign, IL: AOCS Press. 
Crosby AJ, Wahle KWJ \& Duthie GG (1996) Modulation of glutathione peroxidase activity in human vascular endothelial cells by fatty acids and the cytokine interleukin-1ß. Biochimica et Biophysica Acta 1303, 187-192.

Davidson J \& Rotondo D (2000) Lipid Metabolism. Current Opinion in Lipidology 11, 211-213.

De Caterina R, Cybulsky MI, Clinton SK, Gimbrone MA \& Libby P (1994) The omega-3 fatty acid docosahexaenoate reduces cytokine-induced expression of proatherogenic and proinflammatory proteins in humans. Arteriosclerosis and Thrombosis 14, 1829-1836.

De Caterina R \& Libby P (1996) Control of endothelial leukocyte adhesion molecules by fatty acids. Lipids 31, S57-S63.

Deglon N, Wilson A, Desponds C, Laurent P, Bron C \& Fasel N (1995) Fatty acids regulate Thy-lantigen mRNA stability in T-lymphocyte precursors. European Journal of Biochemistry 231, 687-696.

Duplus E, Glorian M \& Forest C (2000) Fatty acid regulation of gene transcription. Journal of Biological Chemistry 275, 30749-30752.

Farquharson A, Wu H-C, Grant I, Choung J-J, Eremin O, Heys SD \& Wahle KWJ (1999) Possible mechanisms for the putative anti-atherogenic and anti-tumorigenic effects of conjugated polyenoic fatty acids. Lipids $\mathbf{3 4}, \mathrm{S} 343$.

Finstad HS, Kolset SO, Holme JA, Wiger R, Farrants AK, Blomhoff R \& Drevon CA (1994) Effect of $n-3$ and $n-6$ fatty acids on proliferation and differentiation of promyelocytic leukemic HL-60 cells. Blood 14, 191-197.

Flick PK, Chen J \& Vagelos PR (1977) Effect of dietary polyunsaturated fatty acids on the activity and content of fatty acid synthetase from rat liver. Journal of Biological Chemistry 252, 4242-4249.

Forest C, Franckhauser S, Glorian M, Antras-Ferry J, Robin D \& Robin P (1997) Regulation of gene transcription by fatty acids, fibrates and prostaglandins: the phosphoenolpyruvate carboxykinase gene as a model. Prostaglandins, Leukotrienes and Essential Fatty Acids 57, 47-56.

Funk CD (2001) Prostaglandins and leukotrienes: advances in eicosanoid biology. Science 294, 1871-1875.

Grimble RF, Howell WM, O'Reilly G, Turner SJ, Markovic O, Hirrell S, East JM \& Calder PC (2002) The ability of fish oil to suppress tumour necrosis factor alpha production by peripheral blood mononuclear cells in healthy men is associated with polymorphisms in genes that influence tumour necrosis factor alpha production. American Journal of Clinical Nutrition 76, 454-459.

Gurr MI \& Harwood JL (1991) Lipid Biochemistry: An Introduction. London: Chapman \& Hall.

Haddad JJ, Saade NE \& Safieh-Garabedian B (2002) Redox regulation of TNF-alpha biosynthesis: augmentation by irreversible inhibition of gamma-glutamylcysteine synthetase and the involvement of an IkappaB-alpha/NF-kappaBindependent pathway in alveolar epithelial cells. Cell Signalling 14, 211-218.

Huang TT, Kudo N, Yoshida M \& Miyamoto S (2000) A nuclear export signal in the $\mathrm{N}$-terminal regulatory domain of IкB- $\alpha$ controls cytoplasmic localisation of inactive NF- $\kappa \mathrm{B} / \mathrm{I} \kappa \mathrm{B}$ complexes. Proceedings of the National Academy of Sciences USA 97, 1014-1019.

Hughes DA \& Pinder AC (1997) N-3 polyunsaturated fatty acids modulate the expression of functionally associated molecules on human monocytes and inhibit antigen presentation in vitro. Clinical and Experimental Immunology 110, 516-523.

Hughes DA, Pinder AC, Piper Z, Johnston IT \& Lund EK (1996) Fish oil supplementation inhibits the expression of major histocompatibility complex class II molecules and adhesion molecules on human monocytes. American Journal of Clinical Nutrition 63, 267-272.
Ip C, Briggs SP, Haegele AD, Thompson HJ, Stokson J \& Scimeca JA (1996) The efficacy of conjugated linoleic acid in mammary cancer prevention is independent of the level and type of fat in the diet. Carcinogenesis 17, 1045-1050.

Ip C, Ip MM, Loftus T, Shoemaker S \& Shea-Eaton W (2001) Induction of apoptosis by conjugated linoleic acid in cultured mammary tumor cells and pre-malignant lesions of the rat mammary gland. Cancer Epidemiology, Biomarkers and Prevention 9, 689-696.

Ip C, Scimeca JA \& Thompson HJ (1995) Effect of timing and duration of dietary conjugated linoleic acid on mammary cancer prevention. Nutrition and Cancer 24, 241-247.

Jeffcoat R \& James AT (1978) The control of stearoyl-CoA desaturase by dietary linoleic acid. FEBS Letters 85, 114-118.

Jones BH, Maher MA, Banz WJ, Zemel MB, Whelan J, Smith PJ \& Moustaid N (1996) Adipose tissue stearoyl-CoA desaturase mRNA is increased by obesity and decreased by polyunsaturated fatty acids. American Journal of Physiology 271, E44-E49.

Jump DB \& Clarke SD (1999) Regulation of gene expression by dietary fat. Annual Review of Nutrition 19, 63-90.

Jump DB, Clarke SD, MacDougald OA \& Thelen A (1993) Polyunsaturated fatty acids inhibit S14 gene transcription in rat liver and cultured hepatocytes. Proceedings of the National Academy of Sciences USA 90, 8454-8458.

Kersten S, Desvergne B \& Wahli W (2000) Roles of PPAR's in health and disease. Nature 405, 421-424.

Kliewer SA, Sundseth SS, Jones SA, Brown PJ, Wisely GB, Koble CS, Devchand P, Wahli W, Willson TM, Lenhard JM \& Lehmann JM (1997) Fatty acids and eicosanoids regulate gene expression through direct interaction with peroxisome proliferator-activated receptors $\alpha$ and $\gamma$. Proceedings of the National Academy of Sciences USA 94, 4318-4323.

Kritchevsky D (1999) Conjugated linoleic acid and experimental atherosclerosis in rabbits. In Advances in Conjugated Linoleic Acid Research, vol. 1, pp. 397-403 [MP Yurawecz, MM Mossoba, JKG Kramer, MW Pariza and GJ Nelson, editors]. Champaign, IL: AOCS Press.

Landschulz KT, Jump DB, MacDougald OA \& Lane MD (1994) Transcriptional control of the stearoyl-CoA desaturase-1 gene by polyunsaturated fatty acids. Biochemical and Biophysical Research Communications 200, 763-768.

Majumder B, Wahle KWJ, Moir S, Schofiels AC, Choe S-N, Farquharson A, Grant I \& Heys SD (2002) Conjugated linoleic acids (CLA's) regulate the expression of key apoptotic genes in human breast cancer cells. FASEB Journal available at: www.fasebj.org/cgi/doi/10.1096/fj.01-0720fje

Meydani SN, Endres S, Woods MM, Goldin BR, Soo C, MorrillLabrode A, Dinarello CA \& Gorbach SL (1991) Oral (n-3) fatty acid supplementation suppresses cytokine production and lymphocyte proliferation: comparison between young and older women. Journal of Nutrition 121, 547-555.

Moya-Camarena SY, Vanden heuvel JP, Blanchard SG, Leesnitzer LA \& Belury MA (1999) Conjugated linoleic acid is a potent naturally occurring ligand and activator of PPAR-alpha. Journal of Lipid Research 40, 1426-1433.

Mustapha S, Kirshner A, De Moissac D \& Kirschenbaum LA (2000) A direct requirement of nuclear factor kappa B for suppression of apoptosis in ventricular myocytes. American Journal of Physiology 279, H939-H945.

Natarajan R, Ghosh S, Fisher BJ, Diegelmann RF, Wiley A, Walsh S, Graham MF \& Fowler AA (2001) Redox imbalance in Crohn's disease intestinal smooth muscle cells causes NF-kappa B-mediated spontaneous interleukin-8 secretion. Journal of Interferon and Cytokine Research 21, 349-359.

Niot I, Poirier H \& Besnard P (1997) Regulation of gene expression by fatty acids: special reference to fatty acid binding protein (FABP). Biochemie 79, 129-133. 
Ntambi JM (1992) Dietary regulation of stearoyl-CoA desaturase-1 gene expression in mouse liver. Journal of Biological Chemistry 267, 10925-10930.

Ou J, Tu H, Shan B, Luk A, DeBose-Boyd RA, Bashmakov Y, Goldstein JL \& Brown MS (2001) Unsaturated fatty acids inhibit transcription of the sterol regulatory element binding protein-1c (SREBP-1c) gene by antagonising ligand-dependent activation of the LXR. Proceedings of the National Academy of Sciences USA 98, 6027-6032.

Pariza MW (1999) The biological activities of conjugated linoleic acid. In Advances in Conjugated Linoleic Acid Research, vol. 1, pp. 12-20 [MP Yurawecz, MM Mossoba, JKG Kramer, MW Pariza and GJ Nelson, editors]. Champaign, IL: AOCS Press.

Petrova TV, Akama KT \& Van Eldik LJ (1999) Cyclopentenone prostaglandins suppress activation of microglia: down-regulation of inducible nitric-oxide synthase by 15 -deoxy- $\Delta^{12,14}$ prostaglandin $\mathrm{J}_{2}$. Proceedings of the National Academy of Sciences USA 96, 4668-4673.

Raclot T, Groscolas R, Langin D \& Ferre P (1997) Site-specific regulation of gene expression by $\mathrm{n}-3$ polyunsaturated fatty acids in rat white adipose tissues. Journal of Lipid Research 38, 1963-1972.

Reseland JE, Anderssen SA, Solvoll K, Hjerman I, Urdal P, Holme I \& Drevon CA (2001a) Effect of long-term changes in diet and exercise on plasma leptin concentrations. American Journal of Clinical Nutrition 73, 240-245.

Reseland JE, Haugen F, Hollung K, Solvoll K, Halvorsen B, Brude IR, Nenseter MS, Christiansen ER \& Drevon CA (2001b) Reduction of leptin gene expression by dietary polyunsaturated fatty acids. Journal of Lipid Research 42, 743-750.

Robinson DR, Knoell CT, Urakaze M, Huang R, Taki H, Sugiyama E, Xu L-L, Yeh ETH, Olesiak W, Guo M, Colvin RB \& Auron PE (1995) Suppression of autoimmune disease by $\omega-3$ fatty acids. Biochemical Society Transactions 23, 287-291.

Rossi A, Elia G \& Santoro MG (1997) Inhibition of nuclear factor $\kappa \mathrm{B}$ by prostaglandin $\mathrm{A}_{1}$ : an effect associated with heat shock transcription factor activation. Proceedings of the National Academy of Sciences USA 94, 746-750.

Rotondo D (1995) Fatty acid modulation of cell responsiveness. Biochemical Society Transactions 23, 291-296.

Rotondo D \& Davidson J (2002) Prostaglandins and PPAR control immune cell function. Immunology 105, 20-22.

Rozera C, Carattoli A, De Marco A, Amici C, Giorgi C \& Santoro MG (1996) Inhibition of HIV-1 replication by cyclopentenone prostaglandins in acutely infected human cells: evidence for a transcriptional block. Journal of Clinical Investigation 97, 1795-1803.

Salati LM \& Clarke SD (1986) Fatty acid inhibition of hormonal induction of acetyl-Co enzyme A carboxylase in hepatocyte monolayers. Archives of Biochemistry and Biophysics 246, 8290.

Schwartz RS \& Abraham S (1982) Effect of dietary polyunsaturated fatty acids on the activity and content of fatty acid synthetase in mouse liver. Biochimica et Biophysica Acta 221, 206-215.
Sessler AM \& Ntambi JM (1998) Polyunsaturated fatty acid regulation of gene expression. Journal of Nutrition 128, 923-926.

Song HJ, Yu G, Bestwick CS, Sneddon AA, Choe S-N, Grant I, Heys SD \& Wahle KWJ (2003) Fatty acid regulation of NF- $\kappa B$ activation in different types of prostate cancer cells. Biochemical and Biophysical Research Communications (In the Press).

Straus DS, Pascual G, Li M, Welch JS, Ricote M, Hsiang C-H, Sengchanthalangsy LL, Ghosh G \& Glass CK (2000) 15-Deoxy$\Delta^{12,14}$-prostaglandin $\mathrm{J}_{2}$ inhibits multiple steps in the NF- $\mathrm{BB}$ signalling pathway. Proceedings of the National Academy of Sciences USA 97, 4844-4849.

Sugano M, Yamasaki M, Yamada K \& Huang Y-S (1999) Effect of conjugated linoleic acids on polyunsaturated fatty acid metabolism and immune function. In Advances in Conjugated Linoleic Acid Research, vol. 1, pp. 1327-339 [MP Yurawecz, MM Mossoba, JKG Kramer, MW Pariza and GJ Nelson, editors]. Champaign, IL: AOCS Press.

Tebbey PW \& Buttke TM (1993) Independent arachidonic acid-mediated gene regulatory pathways in lymphocytes. Biochemical and Biophysical Research Communications 194, 862-868.

Tebbey PW, Van Cleave S \& Buttke TM (1994) Induction of stearoyl-CoA desaturase 2 gene expression correlates with fatty acid changes in phosphatidylcholine. Biochemistry and Molecular Biology International 33, 991-1000.

Wahle KWJ, Farquharson A, Hesketh J \& Thompson MG (1997) Induction of mRNA expression for glutathione peroxidase (GPx) in HUVEC by cytokines and polyunsaturated fatty acids. Chemistry and Physics of Lipids 88, 136-137.

Wahle KWJ \& Heys SD (2002) Cell signal mechanisms, conjugated linoleic acids (CLA's) and anti-tumorigenesis. Prostaglandins, Leukotrienes and Essential Fatty Acids 67, 183-186.

Wahle KWJ \& Radcliffe JD (1977) Effect of a diet rich in sunflower oil on aspects of lipid metabolism in genetically obese rats. Lipids 12, 135-139.

Wahle KWJ \& Rotondo D (1999) Fatty acids and endothelial cell function: regulation of adhesion molecule and redox enzyme expression. Current Opinion in Clinical Nutrition and Metabolic Care 2, 109-115.

Witztum JL \& Steinberg D (2001) The oxidative modification hypothesis of atherosclerosis: does it hold for humans? Trends in Cardiovascular Medicine 11, 93-102.

Wu H-C, Arthur JR \& Wahle KWJ (1998) Regulation of phospholipid glutathione peroxidase (PHGPx) gene expression in human endothelial cells. Chemistry and Physics of Lipids 94, 174.

Yaqoob P (1998) Lipids and the immune response. Current Opinion in Clinical Nutrition and Metabolic Care 1, 153-161.

Yu Y, Correll PH \& Vanden Heuvel JP (2002) Conjugated linoleic acid decreases production of pro-inflammatory products in macrophages: evidence for a PPAR- $\gamma$-dependent mechanism. Biochimica et Biophysica Acta 1581, 89-99. 